\title{
Multiple endocrine dysfunctions in a patient with secondary hemochromatosis
}

Ibtissem Oueslati, Emna Elfeleh, Karima Khiari, Nejib Ben Abdallah.

Department of Endocrinology, Charles Nicolle Hospital. Tunis, Tunisia.

\section{Introduction:}

Hemochromatosis is a disorder caused by an excess of iron deposition in the parenchymal cells that leads to organ dysfunction. In patients with secondary hemochromatosis due to multiple blood transfusions, endocrinopathies such as diabetes mellitus, hypopituitarism frequently develop.

Herein we report the case of a patient with B-thalassemia major who developed diabetes mellitus, hypopituitarism and primary hypoparathyroidism due to secondary hemochromatosis .

\section{Case report:}

A 27-year-old male patient was diagnosed at the age of 4 years with hemochromatosis secondary to multiple transfusions for B-thalassemia major.

At the age of 19 years, he developed diabetes mellitus and hypopituitarism with hypogonadotropic hypogonadism and corticotropin deficiency. MRI showed hemosiderin deposits in anterior pituitary gland. He received insulin, hydrocortisone and testosterone enanthate. One year later, he was diagnosed as having iron-overload cardiomyopathy and hepatic cirrhosis.

At the age of 27 years, the patient presented with a weakness. On physical examination, he had a body weight of $56 \mathrm{~kg}$, a body mass index of $17.2 \mathrm{~kg} / \mathrm{m}^{2}$, a blood pressure of $90 / 60 \mathrm{mmHg}$ and a pulse rate of 75/mn.

Laboratory tests indicated a central hypothyroidism with a TSH of $3.94 \mu \mathrm{IU} / \mathrm{ml}$ and FT4 of $0.66 \mathrm{ng} / \mathrm{ml}$ and a hypoparathyroidism with hypocalcemia (corrected calcemia of $2.05 \mathrm{mmol} / \mathrm{l})$, hyperphosphatemia (1.91 $\mathrm{mmol} / \mathrm{l})$ and low PTH level (13.3 pg/ml).

He was treated with levothyroxine and 1 a hydroxyvitamin D3.

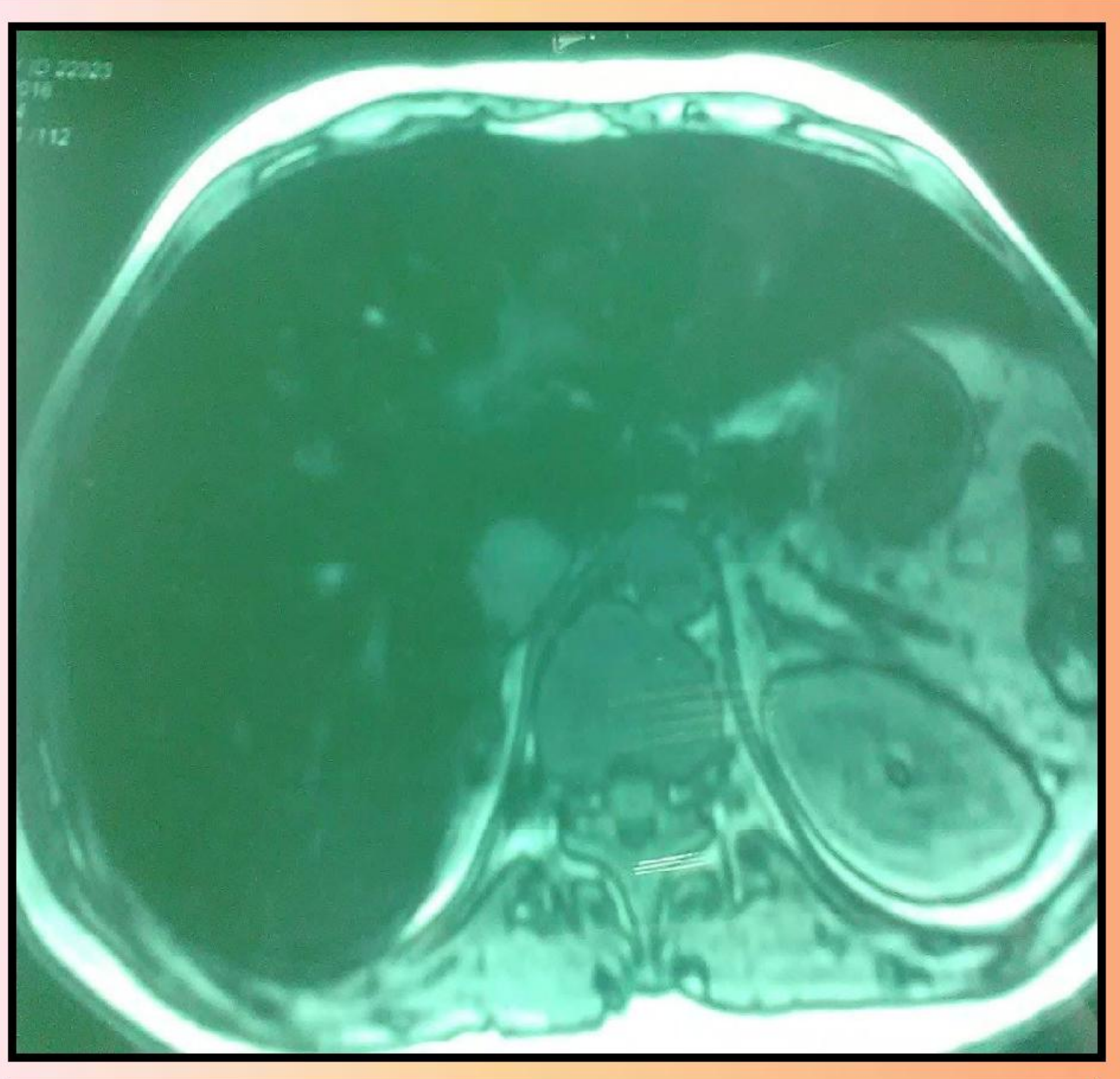

liver parenchyma in T2 hypointense related to iron overload

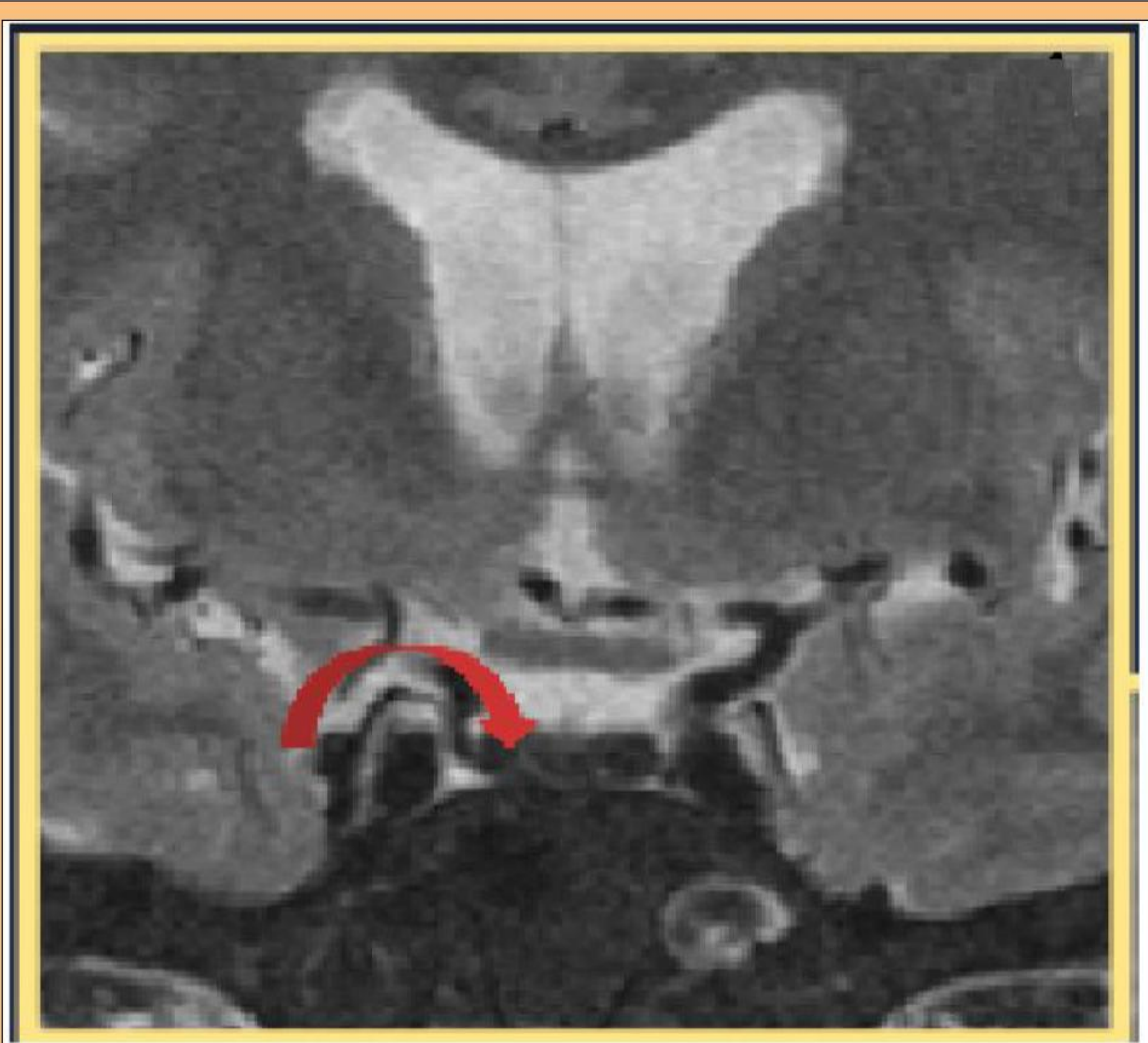

Anterior pituitary in T2 hypointense indicating iron overload

\section{Conclusion:}

Patients with secondary hemochromatosis due to repeated transfusions may develop irreversible multiple endocrine failures. Therefore, a regular use of chelation therapy and a follow up with repeated screening examinations are necessary in patients with multiple transfusions . 